\title{
Cordial and 3-Equitable Labeling for Some Shell Related Graphs
}

\author{
S. K. Vaidya ${ }^{1}$, N. A. Dani, K. K. Kanani, and P. L. Vihol \\ Department of Mathematics, Saurashtra University, Rajkot-360005, Gujrat, India \\ Received 27 March 2009, accepted in revised form 18 June 2009
}

\begin{abstract}
We present here cordial and 3-equitable labeling for the graphs obtained by joining apex vertices of two shells to a new vertex. We extend these results for $k$ copies of shells.

Keywords: Cordial labeling; 3-equitable labeling; Shell.

(c) 2009 JSR Publications. ISSN: 2070-0237 (Print); 2070-0245 (Online). All rights reserved.

DOI: 10.3329/jsr.v1i3.2227

J. Sci. Res. 1 (3), 438-449 (2009)
\end{abstract}

\section{Introduction}

We begin with simple, finite, connected and undirected graph $G=(V, E)$. For all standard terminology and notations we follow Harary [1]. We will give brief summary of definitions which are useful for the present investigations.

Definition 1.1 A shell $S_{n}$ is the graph obtained by taking $n$-3 concurrent chords in a cycle $C_{n}$ on $n$ vertices. The vertex at which all the chords are concurrent is called the apex vertex. The shell is also called fan $F_{n-1}$. i.e. $S_{n}=F_{n-1}=P_{n-1}+\mathrm{K}_{1}$.

Definition 1.2: Consider two shells $S_{n}{ }^{(1)}$ and $S_{n}{ }^{(2)}$ then graph $G=<S_{n}{ }^{(1)}: S_{n}{ }^{(2)}>$ obtained by joining apex vertices of shells to a new vertex $x$.

Definition 1.3: Consider k copies of shells namely $S_{n}{ }^{(1)}, S_{n}{ }^{(2)}, S_{n}{ }^{(3)}, \ldots, S_{n}{ }^{(k)}$. Then the graph $G=<S_{n}{ }^{(1)}: S_{n}{ }^{(2)}: S_{n}{ }^{(3)}: \ldots: S_{n}{ }^{(k)}>$ obtained by joining apex vertex of each $S_{n}{ }^{(p)}$ and apex of $S_{n}^{(p-1)}$ to a new vertex $x_{p-1}$ where $2 \leq p \leq k$.

Definition 1.4: If the vertices of the graph are assigned values subject to certain conditions then it is known as graph labeling.

Most interesting graph labeling problems have following three important ingredients.

(i) a set of numbers from which the labels are chosen;

(ii) a rule that assigns a value to each edges;

(iii) a condition that these values must satisfy.

${ }^{1}$ Corresponding Author : samirkvaidya@yahoo.co.in 
For detail survey on graph labeling one can refer Gallian [2]. Vast amount of literature is available on different types of graph labeling. According to Beineke and Hegde [3] graph labeling serves as a frontier between number theory and structure of graphs. Labeled graph have variety of applications in coding theory, particularly for missile guidance codes, design of good radar type codes and convolution codes with optimal autocorrelation properties. Labeled graph plays vital role in the study of X-ray crystallography, communication network and to determine optimal circuit layouts. A detail study of variety of applications of graph labeling is given by Bloom and Golomb[4].

Definition 1.5: Let $G=(V, E)$ be a graph. A mapping $f: V(G) \rightarrow\{0,1\}$ is called binary vertex labeling of $G$ and $f(v)$ is called the label of the vertex $v$ of $G$ under $f$.

For an edge $e=u v$, the induced edge labeling $f^{*}: E(G) \rightarrow\{0,1\}$ is given by $f^{*}(e)=\mid f$ $(u)-f(v) \mid$. Let $v_{f}(0), v_{f}(1)$ be the number of vertices of $G$ having labels 0 and 1 respectively under $f$ while $e_{f}(0), e_{f}(1)$ be the number of edges having labels 0 and 1 respectively under $f^{*}$.

Definition 1.6: A binary vertex labeling of a graph $G$ is called a cordial labeling if $\mid v_{f}(0)$ $-v_{f}(1) \mid \leq 1$ and $\left|e_{f}(0)-e_{f}(1)\right| \leq 1$. A graph $G$ is cordial if it admits cordial labeling.

The concept of cordial labeling was introduced by Cahit [5]. Many researchers have studied cordiality of graphs. Cahit [5] proved that tree is cordial. In the same paper he proved that $K_{n}$ is cordial if and only if $n \leq 3$. Ho et al. [6] proved that unicyclic graph is cordial unless it is $C_{4 k+2}$. Andar et al. [7] have discussed the cordiality of multiple shells. Vaidya et al. [8, 9, 10] have also discussed the cordiality of various graphs.

Definition 1.7: Let $G=(V, E)$ be a graph. A mapping $f: V(G) \rightarrow\{0,1,2\}$ is called ternary vertex labeling of $G$ and $f(v)$ is called the label of the vertex $v$ of $G$ under $f$.

For an edge $e=u v$, the induced edge labeling $f^{*}: E(G) \rightarrow\{0,1,2\}$ is given by $f^{*}(e)=\mid f$ $(u)-f(v) \mid$. Let $v_{f}(0), v_{f}(1)$ and $v_{f}(2)$ be the number of vertices of $G$ having labels 0,1 and 2 respectively under $f$ while $e_{f}(0), e_{f}(1)$ and $e_{f}(2)$ be the number of edges having labels 0,1 and 2 respectively under $f^{*}$.

Definition 1.8: A vertex labeling of a graph $G$ is called a 3-equitable labeling if $\mid v_{f}(i)$ $-v_{f}(j) \mid \leq 1$ and $\left|e_{f}(i)-e_{f}(j)\right| \leq 1$ for all $0 \leq i, j \leq 2$. A graph $G$ is 3-equitable if it admits 3equitable labeling.

The concept of 3-equitable labeling was introduced by Cahit [11]. Many researchers have studied 3-equitability of graphs. For example Cahit [11] proved that $C_{n}$ is 3-equitable except $n \equiv 3(\bmod 6)$. In the same paper he proved that an Eulerian graph with number of edges congruent to $3(\bmod 6)$ is not 3 -equitable. Youssef [12] proved that $W_{n}$ is 3equitable for all $n \leq 4$. In the present investigations we prove that graphs $\left\langle S_{n}{ }^{(1)}: S_{n}{ }^{(2)}>\right.$ and $<S_{n}{ }^{(1)}: S_{n}{ }^{(2)}: S_{n}{ }^{(3)}: \ldots: S_{n}{ }^{(k)}>$ cordial as well as 3-equitable.

\section{Main Results}

Theorem 2.1: Graph $<S_{n}{ }^{(1)}: S_{n}{ }^{(2)}>$ is cordial. 
Proof: Let $v_{1}{ }^{(1)}, v_{2}{ }^{(1)}, v_{3}{ }^{(1)}, \ldots v_{n}{ }^{(1)}$ be the vertices $S_{n}^{(1)}$ of $v_{1}{ }^{(2)}, v_{2}{ }^{(2)}, v_{3}^{(2)}, \ldots v_{n}{ }^{(2)}$ be the vertices of $S_{n}{ }^{(2)}$. Let $v_{1}{ }^{(1)}$ and $v_{1}{ }^{(2)}$ be the apex vertices of $S_{n}{ }^{(1)}$ and $S_{n}{ }^{(2)}$, respectively. Let $G=<S_{n}{ }^{(1)}: S_{n}{ }^{(2)}>$. We define binary vertex labeling f: $V(G) \rightarrow\{0,1\}$ as follows.

For $j=1,2$

$f\left(v_{i}^{(j)}\right)=0$; if $i \equiv 2,3(\bmod 4)$

$f\left(v_{i}{ }^{(j)}\right)=1$; if $i \equiv 0,1(\bmod 4)$

$f(x) \quad=0$; if $n \equiv 1(\bmod 4)$

$f(x) \quad=1$; if $n=0,2,3(\bmod 4)$

The labeling pattern defined above covers all possible arrangement of vertices. The graph $G$ satisfies the conditions $\left|v_{f}(0)-v_{f}(1)\right| \leq 1$ and $\left|e_{f}(0)-e_{f}(1)\right| \leq 1$. as shown in Table 1 . i.e. $G$ admits cordial labeling.

Let $n=4 a+b, a O N Y\{0\}$.

Table 1 . Table showing vertex and edge conditions.

\begin{tabular}{ccc}
\hline$b$ & Vertex condition & Edge condition \\
\hline $0,1,2$ & $v_{f}(0)+1=v_{f}(1)$ & $e_{f}(0)=e_{f}(1)$ \\
3 & $v_{f}(0)=v_{f}(1)+1$ & $e_{f}(0)=e_{f}(1)$ \\
\hline
\end{tabular}

Illustration 2.2: Consider a graph $G=<S_{7}^{(1)}: S_{7}^{(2)}>$. Here $n=7$. The cordial labeling is as shown in Fig. 1.

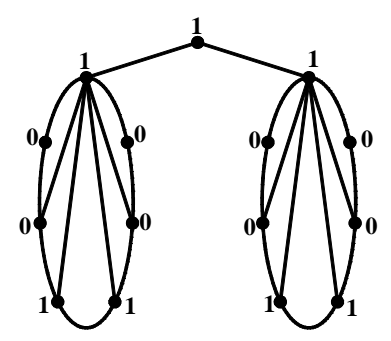

Fig. 1. Cordial labeling of the graph G.

Theorem 2.3: Graph $<S_{n}{ }^{(1)}: S_{n}{ }^{(2)}: S_{n}{ }^{(3)}: \ldots . S_{n}{ }^{(k)}>$ is cordial.

Proof: Let $S_{n}{ }^{(j)}$ be the shells. Let $v_{i}^{(j)}$ be the vertices of $S_{n}{ }^{(j)}$ and $v_{1}{ }^{(j)}$ be the apex vertices of $S_{n}{ }^{(j)}$. Let $x_{j}(j \neq k)$ be the new vertices. Let $G=<S_{n}{ }^{(1)}: S_{n}{ }^{(2)}: S_{n}{ }^{(3)}: \ldots . S_{n}{ }^{(k)}>$. We define binary vertex labeling $f: V(G) \rightarrow\{0,1\}$ as follows.

For $j \equiv 1,2(\bmod 4)$

$f\left(v_{i}^{(j)}\right)=0$; if $i \equiv 2,3(\bmod 4)$

$f\left(v_{i}^{(j)}\right)=1$; if $i=0,1(\bmod 4)$

For $j \equiv 0,3(\bmod 4)$

$f\left(v_{i}^{(j)}\right)=0$; if $i=0,1(\bmod 4)$ 
$f\left(v_{i}^{(j)}\right)=1$; if $i \equiv 2,3(\bmod 4)$

For $n=0,2,3(\bmod 4)$

$f\left(x_{j}\right)=0$; if $j \equiv 2,3(\bmod 4)$

$f\left(x_{j}\right) \quad=1$; if $j \equiv 0,1(\bmod 4), j \neq k$

For $n \equiv 1(\bmod 4)$

$f\left(x_{j}\right)=0$; if $j \equiv 1,2(\bmod 4)$

$f\left(x_{j}\right)=1$; if $j \equiv 0,3(\bmod 4), j \neq k$

The labeling pattern defined above covers all possible arrangement of vertices. The graph $G$ satisfies the conditions $\left|v_{f}(0)-v_{f}(1)\right| \leq 1$ and $\left|e_{f}(0)-e_{f}(1)\right| \leq 1$ as shown in Table 2. i.e. $G$ admits cordial labeling.

Let $n=4 a+b, k=4 c+d, a, c O N Y\{0\}$

Table 2. Table showing vertex and edge conditions.

\begin{tabular}{cccc}
\hline$b$ & $d$ & Vertex condition & Edge condition \\
\hline & 0 & $v_{f}(0)=v_{f}(1)+1$ & $e_{f}(0)=e_{f}(1)$ \\
0 & 1,3 & $v_{f}(0)=v_{f}(1)$ & $e_{f}(0)+1=e_{f}(1)$ \\
& 2 & $v_{f}(0)+1=v_{f}(1)$ & $e_{f}(0)=e_{f}(1)$ \\
\hline & 0 & $v_{f}(0)=v_{f}(1)+1$ & $e_{f}(0)=e_{f}(1)$ \\
& 1 & $v_{f}(0)+1=v_{f}(1)$ & $e_{f}(0)=e_{f}(1)+1$ \\
1 & 2 & $v_{f}(0)+1=v_{f}(1)$ & $e_{f}(0)=e_{f}(1)$ \\
& 3 & $v_{f}(0)=v_{f}(1)+1$ & $e_{f}(0)=e_{f}(1)+1$ \\
\hline \multirow{3}{*}{2} & 0 & $v_{f}(0)=v_{f}(1)+1$ & $e_{f}(0)=e_{f}(1)$ \\
& 1,3 & $v_{f}(0)=v_{f}(1)$ & $e_{f}(0)+1=e_{f}(1)$ \\
& 2 & $v_{f}(0)+1=v_{f}(1)$ & $e_{f}(0)=e_{f}(1)$ \\
\hline \multirow{2}{*}{3} & 0,2 & $v_{f}(0)=v_{f}(1)+1$ & $e_{f}(0)=e_{f}(1)$ \\
& 1,3 & $v_{f}(0)=v_{f}(1)+1$ & $e_{f}(0)+1=e_{f}(1)$ \\
\hline
\end{tabular}

Illustration 2.4: Consider a graph $G=<S_{5}^{(1)}: S_{5}^{(2)}: S_{5}^{(3)}>$. Here $n=5$. The cordial labeling is as shown in Fig. 2.

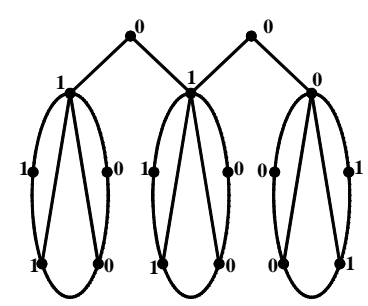

Fig. 2. Cordial labeling of the graph G. 
Theorem 2.5: Graph $<S_{n}{ }^{(1)}: S_{n}{ }^{(2)}>$ is 3-equitable.

Proof: Let $\mathrm{v}_{1}{ }^{(1)}, v_{2}{ }^{(1)}, \ldots v_{n}{ }^{(1)}$ be the vertices $S_{n}{ }^{(1)}$ and $v_{1}{ }^{(2)}, v_{2}{ }^{(2)}, v_{3}{ }^{(2)}, \ldots v_{\mathrm{n}}{ }^{(2)}$ be the vertices $S_{n}^{(2)}$. Let $v_{1}{ }^{(1)}$ and $v_{1}{ }^{(2)}$ be the apex vertices of $S_{n}{ }^{(1)}$ and $S_{n}{ }^{(2)}$, respectively. Let $G$ $=<S_{n}{ }^{(1)}: S_{n}{ }^{(2)}>$. We define ternary vertex labeling $f: V(G) \rightarrow\{0,1,2\}$ as follows.

Case-1: For $n \equiv 0,5(\bmod 6)$

$f\left(v_{i}^{(1)}\right)=0$; if $i \equiv 1,4(\bmod 6)$ $f\left(v_{i}^{(1)}\right)=1$; if $i \equiv 0,5(\bmod 6)$ $f\left(v_{i}{ }^{(1)}\right)=2$; if $i \equiv 2,3(\bmod 6)$ $f\left(v_{i}^{(2)}\right)=0$; if $i \equiv 0,3(\bmod 6)$ $f\left(v_{i}^{(2)}\right)=1$; if $i \equiv 4,5(\bmod 6)$ $f\left(v_{i}^{(2)}\right)=2$; if $i \equiv 1,2(\bmod 6)$ $f(x)=0$;

Case-3: For $n \equiv 2(\bmod 6)$ $f\left(v_{i}^{(1)}\right)=0$; if $i \equiv 1,4(\bmod 6), i \neq n-1$ $f\left(v_{i}^{(1)}\right)=1$; if $i=0,5(\bmod 6)$ $f\left(v_{i}^{(1)}\right)=2$; if $i \equiv 2,3(\bmod 6), i \neq n$ $f\left(v_{i}^{(2)}\right)=0$; if $i \equiv 0,3(\bmod 6)$ $f\left(v_{i}^{(2)}\right)=1 ;$ if $i \equiv 4,5(\bmod 6)$ $f\left(v_{i}^{(2)}\right)=2$; if $i \equiv 1,2(\bmod 6), i \neq n$ $f\left(v_{n-1}{ }^{(1)}\right)=1$;

$f\left(v_{n}{ }^{(1)}\right)=f\left(v_{n}{ }^{(2)}\right)=0$;

$f(x)=2$;
Case-2: For $n \equiv 1(\bmod 6)$

$f\left(v_{i}^{(1)}\right)=0$; if $i \equiv 1,4(\bmod 6), i \neq n$ $f\left(v_{i}^{(1)}\right)=1$; if $i \equiv 0,5(\bmod 6)$ $f\left(v_{i}^{(1)}\right)=2$; if $i \equiv 2,3(\bmod 6)$ $f\left(v_{n}{ }^{(1)}\right)=1$; $f\left(v_{i}^{(2)}\right)=0$; if $i \equiv 0,3(\bmod 6)$ $f\left(v_{i}^{(2)}\right)=1$; if $i \equiv 4,5(\bmod 6)$ $f\left(v_{i}^{(2)}\right)=2$; if $i \equiv 1,2(\bmod 6)$ $f(x)=0$;

Case-4: For $n \equiv 3(\bmod 6)$

$f\left(v_{i}^{(1)}\right)=0$; if $i \equiv 1,4(\bmod 6), i \neq n-2$ $f\left(v_{i}^{(1)}\right)=1$; if $i=0,5(\bmod 6)$ $f\left(v_{i}^{(1)}\right)=2$; if $i \equiv 2,3(\bmod 6), i \neq n-1, n$ $f\left(v_{i}^{(2)}\right)=0$; if $i=0,3(\bmod 6), i \neq n$ $f\left(v_{i}^{(2)}\right)=1$; if $i=4,5(\bmod 6)$ $f\left(v_{i}^{(2)}\right)=2$; if $i \equiv 1,2(\bmod 6), i \neq n-1, n-2$ $f\left(v_{n-2}{ }^{(1)}\right)=f\left(v_{n}{ }^{(2)}\right)=1$; $f\left(v_{n-1}{ }^{(1)}\right)=f\left(v_{n-1}{ }^{(2)}\right)=2$; $f\left(v_{n}^{(1)}\right)=f\left(v_{n-2}{ }^{(2)}\right)=0$; $f(x) \quad=0$;

Case-5: For $n \equiv 4(\bmod 6)$

$f\left(v_{i}^{(1)}\right)=0$; if $i \equiv 1,4(\bmod 6)$

$f\left(v_{i}^{(1)}\right)=1$; if $i=0,5(\bmod 6)$

$f\left(v_{i}^{(1)}\right)=2$; if $i \equiv 2,3(\bmod 6)$

$f\left(v_{i}^{(2)}\right)=0$; if $i \equiv 0,3(\bmod 6), i \neq n-1$

$f\left(v_{i}^{(2)}\right)=1$; if $i \equiv 4,5(\bmod 6)$

$f\left(v_{i}^{(2)}\right)=2$; if $i \equiv 1,2(\bmod 6), i \neq n-2$

$f\left(v_{n-2}{ }^{(2)}\right)=f\left(v_{n-1}{ }^{(2)}\right)=1$;

$f(x) \quad=0$;

The labeling pattern defined above covers all possible arrangement of vertices. The graph $G$ satisfies the conditions $\left|v_{f}(i)-v_{f}(j)\right| \leq 1$ and $\left|e_{f}(i)-e_{f}(j)\right| \leq 1$, where $0 \leq i, j \leq 2$ as shown in Table 3. i.e. $G$ admits 3-equitable labeling.

Let $n=6 a+b, a O N Y\{0\}$. 
Table 3. Table showing vertex and edge conditions.

\begin{tabular}{ccc}
\hline$b$ & Vertex condition & Edge condition \\
\hline 0,3 & $v_{f}(0)=v_{f}(1)+1=v_{f}(2)+1$ & $e_{f}(0)+1=e_{f}(1)=e_{f}(2)$ \\
1,4 & $v_{f}(0)=v_{f}(1)=v_{f}(2)$ & $e_{f}(0)=e_{f}(1)=e_{f}(2)$ \\
2,5 & $v_{f}(0)=v_{f}(1)+1=v_{f}(2)$ & $e_{f}(0)+1=e_{f}(1)+1=e_{f}(2)$ \\
\hline
\end{tabular}

Illustration 2.6: Consider a graph $G=<S_{6}{ }^{(1)}: S_{6}{ }^{(2)}>$. Here $n=6$. The 3-equitable labeling is as shown in Fig. 3.

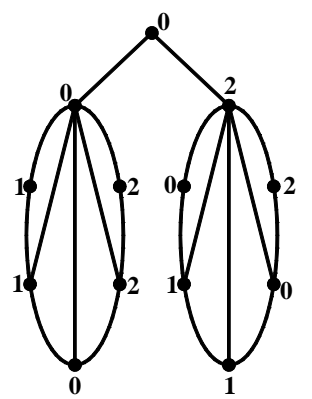

Fig. 3. 3-equitable labeling of the graph G.

Theorem-2.7: Graph $<S_{n}{ }^{(1)}: S_{n}{ }^{(2)}: S_{n}{ }^{(3)}: \ldots: S_{n}{ }^{(k)}>$ is 3-equitable.

Proof: Let $S_{n}{ }^{(j)}$ be the shells. Let $v_{i}^{(j)}$ be the vertices $S_{n}{ }^{(j)}$ and $v_{1}{ }^{(j)}$ be the apex vertices of $S_{n}{ }^{(j)}$. Let $x_{j}(j \neq k)$ be the new vertices. Let $G=<S_{n}{ }^{(1)}: S_{n}{ }^{(2)}: S_{n}{ }^{(3)}: \ldots . S_{n}{ }^{(k)}>$. We define vertex labeling $f: V(G) \rightarrow\{0,1,2\}$ as follows.

Case-1: For $n \equiv 0(\bmod 6)$

Subcase 1.1: $k \equiv 0(\bmod 3)$

For $j \equiv 1(\bmod 3)$

$f\left(v_{i}^{(j)}\right)=0$; if $i \equiv 1,4(\bmod 6)$

For $j \equiv 0,2(\bmod 3)$

$f\left(v_{i}^{(j)}\right)=1$; if $i=0,5(\bmod 6)$

$f\left(v_{i}^{(j)}\right)=0$; if $i \equiv 0,3(\bmod 6) i \neq n$

$f\left(v_{i}{ }^{(j)}\right)=2$; if $i \equiv 2,3(\bmod 6)$

$f\left(v_{i}^{(j)}\right)=1$; if $i \equiv 4,5(\bmod 6)$

$f\left(x_{j}\right)=0$;

$f\left(v_{i}{ }^{(j)}\right)=2$; if $i \equiv 1,2(\bmod 6)$

$f\left(v_{n}{ }^{(j)}\right)=2$; if $j \equiv 2(\bmod 3)$

$f\left(v_{n}{ }^{(j)}\right)=1$; if $j \equiv 0(\bmod 3)$

$f\left(x_{j}\right) \quad=0 ; j \neq k$

Subcase 1.2: $k \equiv 1(\bmod 3)$

For first $k-1$ copies of shells use the pattern of subcase 1.1 and for $k^{\text {th }}$ copy define function as follow.

$f\left(v_{i}^{(k)}\right)=0$; if $i \equiv 1,4(\bmod 6)$

$f\left(v_{i}^{(k)}\right)=1$; if $i \equiv 0,5(\bmod 6)$

$f\left(v_{i}^{(k)}\right)=2$; if $i \equiv 2,3(\bmod 6)$ 
$f\left(x_{k-1}\right)=0$;

Subcase 1.3: $k \equiv 2(\bmod 3)$

For first $k$-2 copies of shells use the pattern of subcase 1.1 and for $k-1$ and $k^{\text {th }}$ copy define function as follow:

$f\left(v_{i}^{(k-1)}\right)=0$; if $i \equiv 1,4(\bmod 6)$

$f\left(v_{i}^{(k-1)}\right)=1$; if $i \equiv 0,5(\bmod 6)$

$f\left(v_{i}^{(k-1)}\right)=2$; if $i \equiv 2,3(\bmod 6)$

$f\left(v_{i}^{(k)}\right)=0$; if $i \equiv 0,3(\bmod 6)$

$f\left(v_{i}^{(k)}\right)=1$; if $i \equiv 4,5(\bmod 6)$

$f\left(v_{i}^{(k)}\right)=2$; if $i \equiv 1,2(\bmod 6)$

$f\left(x_{k-2}\right)=f\left(x_{k-1}\right)=0$;

Case-2: For $n \equiv 1(\bmod 6)$

Subcase 2.1: $k \equiv 0(\bmod 3)$

For $j \equiv 1,2(\bmod 3)$

$f\left(v_{i}^{(j)}\right)=0$; if $i \equiv 1,4(\bmod 6), i \neq n$

For $j \equiv 0(\bmod 3)$

$f\left(v_{i}^{(j)}\right)=1$; if $i \equiv 0,2,3,5(\bmod 6)$ and $j \equiv 1(\bmod 3)$

$f\left(v_{i}^{(j)}\right)=0$; if $i=0,3(\bmod 6)$

$f\left(v_{i}^{(j)}\right)=2$; if $i \equiv 0,2,3,5(\bmod 6)$ and $j \equiv 2(\bmod 3)$

$f\left(v_{i}{ }^{(j)}\right)=1$; if $i \equiv 4,5(\bmod 6)$

$f\left(v_{n}{ }^{(j)}\right)=1$; if $j \equiv 1(\bmod 3)$

$f\left(v_{n}{ }^{(j)}\right)=0$; if $j \equiv 2(\bmod 3)$

$f\left(v_{i}{ }^{(j)}\right)=2$; if $i \equiv 1,2(\bmod 6)$

$f\left(x_{j}\right)=0$; if $j \equiv 1(\bmod 3)$

$f\left(x_{j}\right)=2$; if $j \equiv 2(\bmod 3)$

$f\left(x_{j}\right)=0 ; j \neq k$

Subcase 2.2: $k \equiv 1(\bmod 3)$

For first $k$ - 1 copies of shells use the pattern of subcase 1.1 and for $k^{\text {th }}$ copy define function as follow:

$f\left(v_{i}^{(k)}\right)=0$; if $i \equiv 1,4(\bmod 6)$

$f\left(v_{i}^{(k)}\right)=1$; if $i \equiv 0,5(\bmod 6)$

$f\left(v_{i}^{(k)}\right)=2$; if $i \equiv 2,3(\bmod 6)$

$f\left(x_{k-1}\right)=2$;

Subcase 2.3: $k \equiv 2(\bmod 3)$

For first $k$-2 copies of shells use the pattern of subcase 1.1 and for $k-1$ and $k^{\text {th }}$ copy define function as follow:

For $j=k-1, k$;

$f\left(v_{i}^{(j)}\right)=0$; if $i \equiv 1,4(\bmod 6)$ and $j \neq k, i \neq n$

$f\left(v_{i}^{(j)}\right)=1$; if $i \equiv 0,5(\bmod 6)$

$f\left(v_{i}^{(j)}\right)=2$; if $i \equiv 2,3(\bmod 6)$

$f\left(v_{n}{ }^{(k)}\right)=1$;

$f\left(x_{k-2}\right)=0$;

$f\left(x_{k-1}\right)=2$;

Case-3: For $n \equiv 2(\bmod 6)$

Subcase 3.1: $k \equiv 0(\bmod 3)$ 
For $j \equiv 1(\bmod 3)$

$f\left(v_{i}^{(j)}\right)=0$; if $i \equiv 1,4(\bmod 6)$

$f\left(v_{i}^{(j)}\right)=1$; if $i \equiv 2,3(\bmod 6)$

$f\left(v_{i}^{(j)}\right)=2$; if $i=0,5(\bmod 6)$

$f\left(x_{j}\right)=2$;

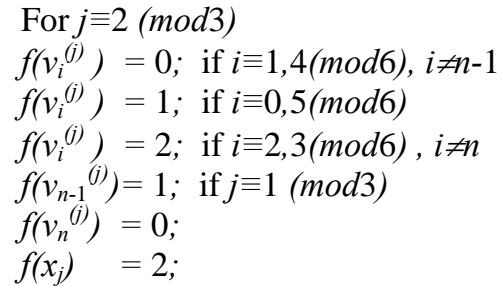

$f\left(v_{i}^{(j)}\right)=0$; if $i \equiv 1,4(\bmod 6), i \neq n-1$

$f\left(v_{i}^{(j)}\right)=1$; if $i=0,5(\bmod 6)$

$f\left(v_{i}^{(j)}\right)=2$; if $i \equiv 2,3(\bmod 6), i \neq n$

$f\left(v_{n}{ }^{(j)}\right)=0$

$f\left(x_{j}\right)=2$;

For $j \equiv 0(\bmod 3)$

$f\left(v_{i}^{(j)}\right)=0$; if $i \equiv 0,5(\bmod 6)$

$f\left(v_{i}^{(j)}\right)=1$; if $i \equiv 2,3(\bmod 6)$

$f\left(v_{i}^{(j)}\right)=2$; if $i \equiv 1,4(\bmod 6)$

$f\left(x_{j}\right) \quad=0 ; j \neq k$

Subcase 3.2: $k \equiv 1(\bmod 3)$

For first $k$-1 copies of shells use the pattern of subcase 1.1 and for $k^{\text {th }}$ copy define function as follow:

$f\left(v_{i}^{(k)}\right)=0$; if $i \equiv 1,4(\bmod 6)$

$f\left(v_{i}^{(k)}\right)=1$; if $i \equiv 2,3(\bmod 6)$

$f\left(v_{i}^{(k)}\right)=2$; if $i=0,5(\bmod 6)$

$f\left(x_{k-1}\right)=2$;

Subcase 3.3: $k \equiv 2(\bmod 3)$

For first $k$-2 copies of shells use the pattern of subcase 1.1 and for $k-1$ and $k^{\text {th }}$ copy define function as follow:

For $j=k-1, k$;

$f\left(v_{i}^{(j)}\right)=0$; if $i \equiv 1,4(\bmod 6)$ and $j \neq k, i \neq 1$

$f\left(v_{i}^{(j)}\right)=1$; if $i \equiv 2,3(\bmod 6)$

$f\left(v_{i}^{(j)}\right)=2$; if $i=0,5(\bmod 6)$

$f\left(v_{1}{ }^{(k)}\right)=2$;

$f\left(x_{k-2}\right)=2$;

$f\left(x_{k-1}\right)=0$;

Case-4: For $n \equiv 3(\bmod 6)$

Subcase 4.1: $k \equiv 0(\bmod 3)$

For $j \equiv 1,2(\bmod 3)$

$f\left(v_{i}^{(j)}\right)=0$; if $i \equiv 1,4(\bmod 6)$

$f\left(v_{i}{ }^{(j)}\right)=1$; if $i \equiv 0,2,3,5(\bmod 6)$ and $j \equiv 2(\bmod 3)$

$f\left(v_{i}{ }^{(j)}\right)=2$; if $i \equiv 0,2,3,5(\bmod 6)$ and $j \equiv 1(\bmod 3)$

$f\left(x_{j}\right)=1$; if $j \equiv 1(\bmod 3)$

$f\left(x_{j}\right)=2$; if $j \equiv 1(\bmod 3)$

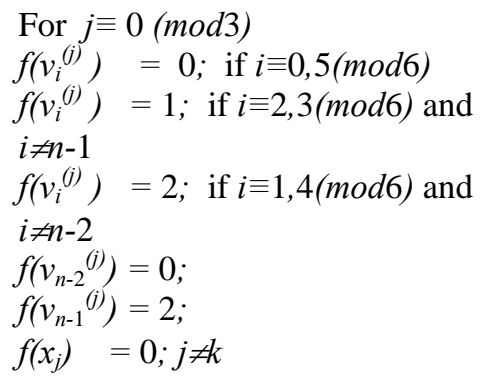

Subcase 4.2: $k \equiv 1(\bmod 3)$ 
For first $k$ - 1 copies of shells use the pattern of subcase 1.1 and for $k^{\text {th }}$ copy define function as follow:

$f\left(v_{i}^{(k)}\right)=0$; if $i \equiv 1,4(\bmod 6)$ and $i \neq n-2$

$f\left(v_{i}^{(k)}\right)=1$; if $i \equiv 2,3(\bmod 6)$ and $i \neq n-1$

$f\left(v_{i}^{(k)}\right)=2$; if $i \equiv 0,5(\bmod 6)$

$f\left(v_{n-2}(k)\right)=2$;

$f\left(v_{n-1}(k)=0\right.$

$f\left(x_{k-1}\right)=0$;

Subcase 4.3: $k \equiv 2(\bmod 3)$

For first $k$-2 copies of shells use the pattern of subcase 1.1 and for $k-1$ and $k^{\text {th }}$ copy define function as follow:

For $j=k-1, k$;

$f\left(v_{i}^{(j)}\right)=0$; if $i \equiv 1,4(\bmod 6)$ and $i \neq n-2, j \neq k-1$

$f\left(v_{i}^{(j)}\right)=1$; if $i \equiv 2,3(\bmod 6)$ and $i \neq n-1$

$f\left(v_{i}{ }^{(j)}\right)=2$; if $i \equiv 0,5(\bmod 6)$

$f\left(v_{n-2}{ }^{(k-1)}\right)=2$;

$f\left(v_{n-1}^{(k-1)}\right)=0$;

$f\left(v_{n-1}{ }^{(k)}\right)=2$;

$f\left(x_{k-2}\right)=f\left(x_{k-1}\right)=0$;

Case-5: For $n \equiv 4(\bmod 6)$

Subcase 5.1: $k \equiv 0(\bmod 3)$

For $j \equiv 1,2(\bmod 3)$

$f\left(v_{i}^{(j)}\right)=0$; if $i \equiv 1,4(\bmod 6)$

For $j \equiv 0(\bmod 3)$

$f\left(v_{i}{ }^{(j)}\right)=1$; if $i \equiv 0,2,3,5(\bmod 6)$ and $j \equiv 2(\bmod 3)$

If $1 \leq i \leq n-4$

$f\left(v_{i}{ }^{(j)}\right)=2$; if $i \equiv 0,2,3,5(\bmod 6)$ and $j \equiv 1(\bmod 3)$

$f\left(v_{i}^{(j)}\right)=0$; if $i \equiv 0,5(\bmod 6)$

$f\left(x_{j}\right)=2$;

$f\left(v_{i}{ }^{(j)}\right)=1$; if $i \equiv 2,3(\bmod 6)$

$f\left(v_{i}^{(j)}\right)=2$; if $i \equiv 1,4(\bmod 6)$

$f\left(v_{n-3}{ }^{(j)}\right)=f\left(v_{n-2}^{(j)}\right)=f\left(v_{n-1}^{(j)}\right)=1$;

$f\left(v_{n}{ }^{(j)}\right)=0$;

$f\left(x_{j}\right) \quad=2 ; j \neq k$

Subcase 5.2: $k \equiv 1(\bmod 3)$

For first $k$ - 1 copies of shells use the pattern of subcase 1.1 and for $k^{\text {th }}$ copy define function as follow:

$f\left(v_{i}^{(k)}\right)=0$; if $i \equiv 1,4(\bmod 6)$ and $i \neq n$

$f\left(v_{i}^{(k)}\right)=1$; if $i \equiv 2,3(\bmod 6)$

$f\left(v_{i}^{(k)}\right)=2$; if $i=0,5(\bmod 6)$

$f\left(v_{n}{ }^{(k)}\right)=1$;

$f\left(x_{k-1}\right)=2$;

Subcase 5.3: $k \equiv 2(\bmod 3)$

For first $k$-2 copies of shells use the pattern of subcase 1.1 and for $k-1$ and $k^{\text {th }}$ copy define function as follow:

$f\left(v_{i}^{(k-1)}\right)=0$; if $i \equiv 1,4(\bmod 6)$ and $i \neq n$

$f\left(v_{i}^{(k-1)}\right)=1$; if $i \equiv 0,5(\bmod 6)$ 
$f\left(v_{i}^{(k-1)}\right)=2 ;$ if $i \equiv 2,3(\bmod 6)$

$f\left(v_{i}^{(k)}\right)=0$; if $i \equiv 1,4(\bmod 6)$ and $i \neq n$

$f\left(v_{i}^{(k)}\right)=1$; if $i \equiv 2,3(\bmod 6)$ and $i \neq n-2$

$f\left(v_{i}^{(k)}\right)=2$; if $i \equiv 0,5(\bmod 6)$

$f\left(v_{n-2}{ }^{(k)}\right)=f\left(x_{k-2}\right)=2$;

$f\left(v_{n}^{(k)}\right)=f\left(v_{n}^{(k-1)}\right)=1$;

$f\left(x_{k-1}\right)=0$;

Case-6: For $n \equiv 5(\bmod 6)$

Subcase 6.1: $k \equiv 0(\bmod 3)$

For $j \equiv 1,2(\bmod 3)$

$f\left(v_{i}^{(j)}\right)=0$; if $i \equiv 1,4(\bmod 6)$

For $j \equiv 0(\bmod 3)$

$f\left(v_{i}{ }^{(j)}\right)=1$; if $i \equiv 0,2,3,5(\bmod 6)$ and $j \equiv 2(\bmod 3)$

If $1 \leq i \leq n-2$

$f\left(v_{i}^{(j)}\right)=2$; if $i \equiv 0,2,3,5(\bmod 6)$ and $j \equiv 1(\bmod 3)$

$f\left(v_{i}^{(j)}\right)=0$; if $i=0,5(\bmod 6)$

$f\left(x_{j}\right)=2$; if $j \equiv 1(\bmod 3)$

$f\left(x_{j}\right)=0 ;$ if $j \equiv 2(\bmod 3)$

$f\left(v_{i}^{(j)}\right)=1$; if $i \equiv 2,3(\bmod 6)$

$f\left(v_{i}^{(j)}\right)=2$; if $i \equiv 1,4(\bmod 6)$

$f\left(v_{n-1}{ }^{(j)}\right)=1$;

$f\left(v_{n}^{(j)}\right)=2$;

$f\left(x_{j}\right) \quad=0 ; j \neq k$

Subcase 6.2: $k \equiv 1(\bmod 3)$

For first $k$ - 1 copies of shells use the pattern of subcase 1.1 and for $k^{\text {th }}$ copy define function as follow:

$f\left(v_{i}^{(k)}\right)=0$; if $i \equiv 1,4(\bmod 6)$

$f\left(v_{i}^{(k)}\right)=1$; if $i \equiv 2,3(\bmod 6)$

$f\left(v_{i}^{(k)}\right)=2$; if $i=0,5(\bmod 6)$

$f\left(x_{k-1}\right)=2$;

Subcase 6.3: $k \equiv 2(\bmod 3)$

For first $k$-2 copies of shells use the pattern of subcase 1.1 and for $k-1$ and $k^{\text {th }}$ copy define function as follow:

$f\left(v_{i}^{(k-1)}\right)=0 ;$ if $i \equiv 1,4(\bmod 6)$

$f\left(v_{i}^{(k-1)}\right)=1$; if $i \equiv 2,3(\bmod 6)$

$f\left(v_{i}^{(k-1)}\right)=2 ;$ if $i \equiv 0,5(\bmod 6)$

$f\left(v_{i}^{(k)}\right)=0$; if $i=0,5(\bmod 6)$

$f\left(v_{i}^{(k)}\right)=1$; if $i \equiv 2,3(\bmod 6)$

$f\left(v_{i}^{(k)}\right)=2$; if $i \equiv 1,4(\bmod 6)$

$f\left(x_{k-2}\right)=2$;

$f\left(x_{k-1}\right)=0$;

The labeling pattern defined above covers all possible arrangement of vertices. The graph $G$ satisfies the conditions $\left|v_{f}(i)-v_{f}(j)\right| \leq 1$ and $\left|e_{f}(i)-e_{f}(j)\right| \leq 1$, where $0 \leq i, j \leq 2$ as shown in Table 4. i.e. $G$ admits 3-equitable labeling. 
Let $n=6 a+b$, and $k=3 c+d$ where $a, c O N Y\{0\}$.

Table 4. Vertex and edge conditions.

\begin{tabular}{cccc}
\hline$b$ & $d$ & Vertex condition & Edge condition \\
\hline & 0 & $v_{f}(0)+1=v_{f}(1)=v_{f}(2)$ & $e_{f}(0)+1=e_{f}(1)=e_{f}(2)+1$ \\
0 & 1 & $v_{f}(0)=v_{f}(1)=v_{f}(2)$ & $e_{f}(0)=e_{f}(1)=e_{f}(2)$ \\
& 2 & $v_{f}(0)=v_{f}(1)+1=v_{f}(2)+1$ & $e_{f}(0)+1=e_{f}(1)=e_{f}(2)+1$ \\
\hline & 0 & $v_{f}(0)+1=v_{f}(1)=v_{f}(2)$ & $e_{f}(0)+1=e_{f}(1)=e_{f}(2)+1$ \\
1 & 1 & $v_{f}(0)+1=v_{f}(1)+1=v_{f}(2)$ & $e_{f}(0)=e_{f}(1)=e_{f}(2)+1$ \\
& 2 & $v_{f}(0)=v_{f}(1)=v_{f}(2)$ & $e_{f}(0)=e_{f}(1)=e_{f}(2)$ \\
\hline 2 & $0,1,2$ & $v_{f}(0)+1=v_{f}(1)=v_{f}(2)$ & $e_{f}(0)+1=e_{f}(1)=e_{f}(2)+1$ \\
\hline & 0 & $v_{f}(0)+1=v_{f}(1)=v_{f}(2)$ & $e_{f}(0)+1=e_{f}(1)=e_{f}(2)+1$ \\
& 1 & $v_{f}(0)=v_{f}(1)=v_{f}(2)$ & $e_{f}(0)=e_{f}(1)=e_{f}(2)$ \\
& 2 & $v_{f}(0)=v_{f}(1)+1=v_{f}(2)+1$ & $e_{f}(0)=e_{f}(1)+1=e_{f}(2)$ \\
\hline & $0(n \neq 4)$ & $v_{f}(0)=v_{f}(1)=v_{f}(2)+1$ & $e_{f}(0)+1=e_{f}(1)=e_{f}(2)+1$ \\
& $0(n=4)$ & $v_{f}(0)=v_{f}(1)=v_{f}(2)+1$ & $e_{f}(0)=e_{f}(1)+1=e_{f}(2)+1$ \\
& 1 & $v_{f}(0)+1=v_{f}(1)+1=v_{f}(2)$ & $e_{f}(0)+1=e_{f}(1)=e_{f}(2)$ \\
& 2 & $v_{f}(0)=v_{f}(1)=v_{f}(2)$ & $e_{f}(0)=e_{f}(1)=e_{f}(2)$ \\
\hline 5 & $0,1,2$ & $v_{f}(0)+1=v_{f}(1)=v_{f}(2)$ & $e_{f}(0)+1=e_{f}(1)=e_{f}(2)+1$ \\
\hline
\end{tabular}

Illustration 2.8: Consider a graph $G=<S_{4}{ }^{(1)}: S_{4}{ }^{(2)}: S_{4}{ }^{(3)}>$. Here $n=4$. The 3-equitable labeling is as shown in Fig. 4.

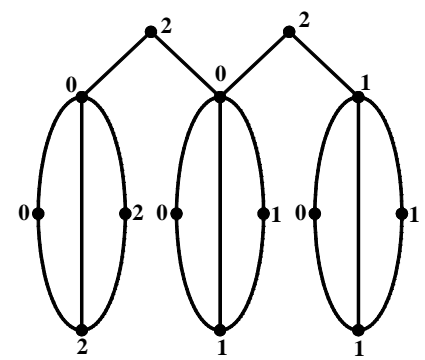

Fig. 4. 3-equitable labeling of the graph G.

\section{Concluding Remarks}

Labeled graph is the topic of current interest for many researchers as it has diversified applications. We discuss here cordial labeling and 3-equitable labeling of some shell related graphs. This approach is novel and contributes four new results. The derived 
labeling pattern is demonstrated by means of elegant illustrations which provide better understanding of the derived results. The results reported here are new and expected to add new dimension to the theory of cordial and 3-equitable graphs.

\section{References}

1. F. Harary, Graph theory (Addison Wesley, Massachusetts, 1972).

2. J. A. Gallian, The Electronics J. of Combinatorics, 16, \#DS6 (2009).

3. L. W. Beineke and S. M. Hegde, Discuss. Math. Graph Theory, 21, 63 (2001).

4. G. S. Bloom and S. W. Golomb, Applications of numbered undirected graphs, Proc of IEEE, 165 (4) (1977) pp. 562-570. doi:10.1109/PROC.1977.10517

5. I. Cahit, Ars Combinatoria 23, 201 (1987).

6. Y. S. Ho, S. M. Lee, and S. C. Shee, Congress. Numer. 68,109 (1989).

7. M. Andar, S. Boxwala and N. B. Limaye, Trends Math.77 (2002).

8. S. K. Vaidya, G. V. Ghodasara, S. Srivastav, and V. J. Kaneria, J. of Indian Math. Society. 76, 237 (2007).

9. S. K. Vaidya, G. V. Ghodasara, S. Srivastav, and V. J. Kaneria, Int. J. of scientific comp. 2 (1), 81 (2008).

10. S. K. Vaidya, S. Srivastav, G. V. Ghodasara, and V. J. Kaneria, Indian J. of Math. and Math. Sc. 4 (2), 145 (2008).

11. I. Cahit, Util. Math. 37, 189 (1990).

12. M. Z. Youssef, Util. Math. 64, 193 (2003). 